Estrategias de intervención

\title{
TEORÍA DE RANGO MEDIO DE ENFERMERÍA PARA LA ADAPTACIÓN DE UN MENOR ANTE DISCRIMINACIÓN Y VIOLENCIA FAMILIAR
}

\author{
NURSING THEORY OF AVERAGE RANGE TO FOR THE ADAPTATION OF A \\ MINOR TO DISCRIMINATION AND FAMILY VIOLENCE
}

\author{
Heidy Urrego Parra \\ Estudiante de Enfermería \\ Universidad Nacional de Colombia, Colombia
}

\section{Viviana Marycel Céspedes Cuevas}

Posdoctorado en Narrativa y Ciencia

Profesora Asociada, Universidad Nacional de Colombia, Sede Bogotá, Facultad de Enfermería, Colombia

Artículo recibido el 21 de junio de 2018. Aceptado en versión corregida el 13 de diciembre de 2018 .

\section{RESUMEN}

El presente artículo desarrolla el proceso de valoración de enfermería en la práctica comunitaria y el análisis de una situación de enfermería en el proceso de adaptación de un menor ante discriminación y violencia familiar. El análisis se realizó desde la teoría de rango medio (TRM) de Afrontamiento de Roy, y se operacionalizó con el proceso de enfermería mostrando que es autónomo, fundamentado y dinámico, ubicada en una visión de mundo integrativa/interactiva; utilizando, además, los patrones de conocimiento de enfermería: empírico, estético, personal y ético. Se expone unas intervenciones y resultados que buscaron mediar el uso de las taxonomías del lenguaje estandarizados, con la meta desde un planteamiento teórico. Se concluye que es fundamental el uso de una TRM en la práctica tanto clínica como comunitaria, que conduzca y guíe la formación del profesional y la forma como se brinda cuidado. Adicionalmente se logra proponer un proceso de enfermería articulado y coherente con la teoría seleccionada, haciendo del presente análisis una estrategia novedosa de gran utilidad para la comprensión e intervención en la práctica, teniendo en cuenta que se parte de un contexto que involucra escuela, familia, hogar y persona que, al recolectar la información de la mayoría los participantes del proceso, se permite comprender y llevar a cabo las intervenciones.

Palabras clave: Adaptación psicológica, Atención de enfermería, Proceso de enfermería, Discriminación, Violencia doméstica. 


\begin{abstract}
This article develops the process of nursing assessment in community practice and the analysis of a nursing situation in the process of adaptation of a child in the face of discrimination and family violence. The analysis was made from Roy's Coping Medium Range Theory (TRM), and it was operationalized with the nursing process showing that it is autonomous, grounded and dynamic, located in an integrative / interactive world view; using, in addition, nursing knowledge patterns: empirical, aesthetic, personal and ethical. Some interventions and results are presented that sought to mediate the use of standardized language taxonomies, with the goal from a theoretical approach. It is concluded that the use of a MRS in clinical and community practice is fundamental, that it guides and guides the professional training and the way care is provided. Additionally, a nursing process articulated and coherent with the selected theory is proposed, making the present analysis a novel strategy of great utility for understanding and intervention in practice, taking into account that it starts from a context that involves school, family, home and person who, by collecting the information of the majority of the participants of the process, is allowed to understand and carry out the interventions.
\end{abstract}

Keywords: Adaptation psychological, nursing care, nursing process, discrimination, domestic violence.

\title{
http://dx.doi.org/10.7764/Horiz_Enferm.29.3.238-250
}

\section{INTRODUCCIÓN}

La práctica de enfermería involucra la vivencia de experiencias con la persona cuidada, generando un proceso de transformación en el cuidado. Es aquí donde se origina la Situación de Enfermería, que se expresa en la presente publicación a través de la narrativa "Un problema con el espejo", siendo el intercambio de emotividades y el reconocimiento de quien ofrece cuidado, el punto medular del análisis; puesto que la enfermera se ve reflejada en la persona cuidada generando la necesidad de cuidarse para de esta forma poder cuidar, y en este caso en particular, generar afrontamiento.

La situación de enfermería se analiza a partir de la Teoría de Rango Medio (TRM) de Afrontamiento de
Callista Roy, derivada de su modelo de adaptación, donde a la persona cuidada, que para este caso es un niño de 5 años afrodescendiente, al cual se le identifican estímulos focales y contextuales como el ser víctima de violencia familiar $\mathrm{y}$ discriminación, para lo cual pueden evidenciarse respuestas adaptativas en distintos ámbitos como el social y conductual que puede ir desde baja autoestima hasta trastornos de atención y de aprendizaje(1), además, se han encontrado efectos en el sistema nervioso central (SNC) que se reflejan en ansiedad por aumento de liberación de noradrenalina crónica, igualmente al estar expuesto a estrés crónico se aumenta la liberación de dopamina reflejándose en inatención, problemas de memoria y 
Teoría de rango medio de enfermería para....

aprendizaje $^{(2)}, \quad$ la impulsividad y agresividad es resultado de la depleción de serotonina $^{(2)}$; todo lo anterior teniendo como base maltrato infantil, discriminación y estrés crónico. Por ello, la importancia de una adecuada valoración de enfermería guiada con una TRM para identificación factores de riesgo y evidenciar signos claves que guíen el cuidado integral del niño junto con la familia, a fin de que los resultados generen una forma eficaz de afrontar y posteriormente la adaptación.

Partiendo de lo anterior se ubica la situación de enfermería en una visión interactiva-integrativa igualmente que la TRM. Se analiza detalladamente el proceso de enfermería desde la postura de Roy, reconociendo los conceptos metaparadigmáticos, los patrones de conocimiento de enfermería y las intervenciones realizadas para iniciar un proceso de afrontamiento en la persona cuidada.

\section{Situación de enfermería: Un problema con el espejo}

Con gran expectativa me dispuse a realizar mi segunda valoración en esta práctica comunitaria llevada a cabo en la Unidad Básica de Atención (U.B.A) en convenio con un colegio público ubicado en el sector; al momento de formar parejas con los niños, decidí hacer equipo con Andrés (El nombre original se ha cambiado para proteger la identidad). Desde el momento que sostuve su mano, por primera vez, la empatía se hizo evidente.

\footnotetext{
En el trayecto del colegio a la U.B.A., mientras conversábamos,
}

pude identificar aquellos estímulos contextuales que influían en el desarrollo de Andrés, por ejemplo, la raza, ser estudiante de transición, el soporte social ineficaz a nivel familiar y escolar y, el conflicto en estos mismos espacios. Además, estímulos focales como: rechazo percibido por su color de piel, la etapa del desarrollo y la disminución visible de la interacción con sus compañeros.

"A los niños les encanta dibujar", teniendo en mente esta afirmación iniciamos la valoración por medio de un dibujo. Mientras Andrés trazaba líneas y hacía figuras me iba relatando su historia, entonces empecé a sospechar de maltrato familiar, racismo y discriminación en la escuela, sin embargo, mi posición fue libre de juicios, respetaba su relato a la vez que ofrecía confianza y prudencia ante cada una de las frases relatadas. Con base en este proceso, pude concluir que el entorno que rodea a Andrés le ofrece estímulos no positivos, donde su yo interno se encuentra vulnerable por no estar lo suficientemente fortalecido, obviamente por su edad; teniendo estrategias de afrontamiento escasas y pocas posibilidades de generar una adecuada adaptación frente a lo ofrecido por su entorno y la etapa del desarrollo del niño.

Por mi cabeza solo rondaba el eco de las palabras racismo y discriminación junto a sensaciones de ira e impotencia. No podía creer que el racismo fuera tan evidente en un salón de clases y cómo una criatura, a tan poca edad, estuviera experimentado tales abusos.

Con el contexto anterior muy presente, seguí realizando la valoración de los aspectos tanto físicos como psicológicos. Entonces evidencié un 
marcado deterioro en la dentición, por la presencia de caries en los segundos molares \#55, 65, 75, 85 y una cavitación en el primer molar \#84. El motivo de este descuido se justificaba en la ausencia de un cepillo de dientes para su uso personal $y$ obviamente en la frecuencia del cepillado que era "de vez en cuando", o mejor dicho, casi nunca. Además, evidencie cicatrices en miembros inferiores de lesiones anteriores, que según lo manifestado por Andrés fueron a causa del castigo con un instrumento al que él llamaba látigo.

Teniendo en cuenta lo mencionado anteriormente, decidí hacer dos actividades; sin embargo, en mi mente rondaban pensamientos de: ;El tiempo es corto!, ;Son tantas las actividades que quiero realizar para trabajarle las estrategias de afrontamiento! que en un momento sentí frustración. Pero aquello no me detiene para llevar a cabo intervenciones como: educación en higiene oral acompañado del obsequio de un cepillo dental y los implementos básicos para una higiene oral adecuada, también, se realizaron actividades para mostrar el cepillado correcto mirándose al espejo. Frente a estas intervenciones, recordaba que, para mí, en el colegio era fastidioso que fuera alguna empresa a promocionar sus productos con el pretexto de dar educación, pero ahora entiendo qué tan importante es incentivar el autocuidado en los niños.

Durante esta la actividad anterior fui feliz cuando Andrés miraba su diente "negro" por la cavitación y me prometía que lo iba a cuidar para que se arreglara. Sin embargo, tuve que remitir a odontología como segunda medida de prevención y corrección. A parte de esto, gracias a nuestra empatía, me hizo una promesa; le dije que debía cuidar su cepillo, que nadie lo debía coger y mucho menos usar, que si era posible lo escondiera, me sonrió y lo prometió.

El segundo momento fue una actividad frente al espejo, donde queríamos percibir el desarrollo y el estado de la autoimagen y el autoconcepto, fundamentales para el desarrollo de estrategia de afrontamiento y finalmente, la adaptación. Tal vez este ejercicio fue complejo para los dos, él no logró mirarse, se inquietó y simplemente quería alejarse, yo en mi esfuerzo por lograr el ejercicio y por mi afán de inspirarle confianza, me desafie y por instantes vencí mi miedo de verme al espejo, esto con el único propósito de que él pudiera trabajar con su yo interno y su identidad.

Tanto él como yo intercambiamos sentimientos, se emocionaba cuando jugábamos, se notaba la felicidad en sus ojos al sentir mi atención, y cuando al oído le repetía que era lindo y que su color de piel era lo más hermoso que tenía, se notaba tímido pero alegre. Yo pude reflexionar a solas luego de haberlo llevado nuevamente a su salón de clase, fue en ese instante cuando comprendi que debía ayudarlo, aportar algo positivo en su vida, que el destino lo había puesto en mi camino para hacerme reaccionar; que el conflicto mío con el espejo es un dilema que necesita solución ahora. Allí entendí que lo más valioso que obtuve de la relación con Andrés fue que mi salud mental debe estar óptima para poder ayudar a quienes necesitan de mi cuidado 
Teoría de rango medio de enfermería para....

y así poder generar un crecimiento mutuo. Es momento de enfrentarme al espejo.

\section{Estrategias de análisis de la situación}

La situación de enfermería " $U n$ problema con el espejo" que nace de un proceso de valoración, se analiza bajo una visión de mundo interactiva-integrativa la cual es coherente con los postulados con la teoría seleccionada, la TRM de Afrontamiento de Roy ${ }^{(3)}$. Es así como se identifican en la situación de enfermería los conceptos metaparadigmáticos y los patrones del conocimiento, a fin de guiar el diseño de las intervenciones y la formulación de los resultados.

\section{Teoría de Rango Medio de Afrontamiento de Callista Roy, aplicada a la Situación de enfermería}

Chinn, Jacobs y Huether ${ }^{(4)}$ determinan que la teoría es "un conjunto de definiciones y proposiciones que proyectan una visión sistemática de un fenómeno, al designarle determinadas relaciones entre los conceptos y que tiene como fin describir, explicar, predecir $\mathrm{y}$ controlar o prescribir el fenómeno". A partir de lo descrito anteriormente es importante resaltar que usar la teoría en la práctica y en la investigación es totalmente viable e innovador; ofreciendo la posibilidad de reconocer conceptos metaparadigmáticos y una visión de mundo que permita generar un cuidado de enfermería dinámico, fundamentado y más autónomo. Es así como se analiza y se selecciona la Teoría de Rango Medio de Afrontamiento de Callista Roy como punto de partida para el análisis de la situación de enfermería. Principalmente al ubicar la teoría y la situación de enfermería en una visión de mundo interactivaintegrativa que ve a la persona como ser holístico en constante relación e interacción con el ambiente.

Desde esta perspectiva el concepto de afrontamiento se define como la manera exitosa de solucionar un evento situacional en el diario vivir. Enfermería constantemente está enfrentada a ver como cada sujeto de cuidado, sea en el ámbito comunitario u hospitalario, utiliza diferentes estrategias de afrontamiento diseñadas por la persona cuidada para finalmente adaptarse a su condición de salud mental y física. Entonces, es pertinente afirmar que la TRM permite su aplicación en la práctica porque su alcance está circunscrito a situaciones de la práctica. Es así, como en 2011 Roy formula las estrategias de afrontamiento que son evaluadas en el proceso de adaptación, y las define como un modelo de formas innatas y adquiridas ${ }^{(5)}$, bajo la influencia directa de estímulos focales y contextuales, que generan una respuesta a los cambios de las situaciones diarias.

Para el caso presentado en la situación de enfermería, se analizan los estímulos focales, contextuales, sus estrategias de afrontamiento y finalmente los resultados (Tabla 1), todo lo anterior articulado con el proceso de enfermería, comprendido como un proceso de afrontamiento eficaz para la adaptación. La generación de estrategias de afrontamiento estuvo enfocada bajo las intervenciones recomendadas por la TRM de Rango Medio de Afrontamiento de Roy las cuales operacionalizaron de forma armónica, las intervenciones y actividades propuestas en la Clasificación de Intervenciones de Enfermería (NIC). 

Tabla 1. Esquema gráfico de la teoría de rango medio de afrontamiento de Roy.

\begin{tabular}{|c|c|c|}
\hline Estímulos focales & $\begin{array}{c}\text { Estrategia de } \\
\text { afrontamiento }\end{array}$ & Resultados \\
\hline $\begin{array}{l}\text { - Disminución de la interacción con sus } \\
\text { iguales. } \\
\text { - Rechazo por el color de piel. } \\
\text { - Abuso verbal percibido por el niño de sus } \\
\text { padres y sus compañeros. } \\
\text { - Etapa del desarrollo. }\end{array}$ & \multirow{3}{*}{$\begin{array}{l}\text { - Evitación pasiva. } \\
\text { - Formas de } \\
\text { cognición perceptiva a } \\
\text { nivel de autoestima y } \\
\text { autoconcepto. } \\
\text { - Generar una } \\
\text { personalidad } \\
\text { extrovertida. }\end{array}$} & \multirow[t]{3}{*}{$\begin{array}{l}\text { Proceso de } \\
\text { afrontamiento } \\
\text { con comporta- } \\
\text { mientos no } \\
\text { adaptativos. }\end{array}$} \\
\hline Estímulos contextuales & & \\
\hline $\begin{array}{l}\text { - Raza y el género } \\
\text { - Conflicto con el soporte social, familiar y } \\
\text { escolar ineficaz. }\end{array}$ & & \\
\hline
\end{tabular}

Fuente: Resultados de la valoración.

Proceso de enfermería: Desde una propuesta de valoración integrada con la teoría de rango medio de afrontamiento de Roy

El proceso de enfermería del cual nace la presente narrativa, está basado en una propuesta de valoración de la salud mental y física, viendo a la persona cuidada desde la teoría, como un ser holístico que constantemente está en interacción con su entorno y frecuentemente utiliza mecanismos innatos $y$ adquiridos para afrontar $y$ adaptarse a los cambios, realizándolo en cuatro modos: fisiológico, autoconcepto, dominio del rol e interdependencia ${ }^{(6)}$. A partir de lo anterior la valoración permitió destacar dos dominios de enfermería que son considerados por los hallazgos los más afectados, siendo estos el dominio de autoconcepto-afrontamiento y el dominio rol-relaciones.

La valoración se realiza en el ámbito físico con la aplicación de indicadores de crecimiento y desarrollo, en el área mental, aplicando instrumentos de tamizaje en salud mental como el RQC y $\mathrm{SRQ}^{(7)}$, para lo cual el primero da positivo y el segundo negativo, además, se evalúa todo el ámbito escolar y familiar con interferencias en este último, por motivos de ausencia de la familia en los procesos escolares de la persona de cuidado (Tabla 2).

Es evidente en la valoración la identificación de un entorno definido como "las condiciones, circunstancias e influencias que rodean y afectan el desarrollo y el comportamiento de los seres humanos como sistemas adaptativos, con particular consideración de la persona 
Teoría de rango medio de enfermería para....

y de los recursos del mundo"(6). De la misma manera, se percibe a la salud claramente afectada, siendo esta un ciclo que permite que una persona tenga las capacidades tanto físicas como mentales para adaptarse y que sus conductas vayan en favor del afrontamiento. Finalmente, se encuentra que la meta de enfermería fue lograr que la persona cuidada buscara la forma ideal de generar afrontamiento a su condición de salud particular con apoyo de la familia; esta meta fue lograda al implementar las intervenciones sugeridas por la TRM, viéndose reflejada en los resultados esperados.

Es importante resaltar que a partir de la valoración y la narrativa se puede dar análisis a los patrones de conocimiento de enfermería. Por ejemplo, el patrón empírico se refleja con la valoración e interpretación de los hallazgos encontrados, que finalmente se vio reflejada en la generación de un plan de cuidados de acuerdo a las necesidades del sujeto. De la misma manera el patrón personal es altamente resaltado e importante para la enfermera, puesto que es quien identifica la necesidad de cuidarse para poder dar cuidado a otros. Para el patrón estético la empatía generada entre la persona cuidada y la enfermera es particular y única, además, la forma de brindar cuidado y de darse cuidado caracterizan esta situación de enfermería. Finalmente, con respecto al patrón ético la prudencia y la confianza ofrecida por la enfermera frente al relato $y$ las manifestaciones del niño, son un aspecto relevante dentro del análisis.

\section{Diagnósticos de enfermería centrales}

Al analizar la valoración bajo la mirada de la TRM de Afrontamiento de Roy, se plantearon dos diagnósticos de enfermería centrales para este caso en particular, obtenidos de la taxonomía Clasificación Completa de la Diagnósticos de Enfermería (NANDA), estos son:

- Riesgo de baja autoestima situacional $\mathrm{R} / \mathrm{C}$ Discriminación y violencia intrafamiliar.

- Deterioro parental $\mathrm{R} / \mathrm{C}$ Violencia intrafamiliar.

Además, a partir de estos, se identifica un diagnóstico secundario, pero igual de importante:

- Deterioro de la dentición $\mathrm{R} / \mathrm{c}$ higiene oral deficiente S/a Deterioro parental.

Durante la valoración se obtuvieron los siguientes datos objetivos y objetivos: 
Teoría de rango medio de enfermería para....

Tabla 2. Recopilación de la valoración.

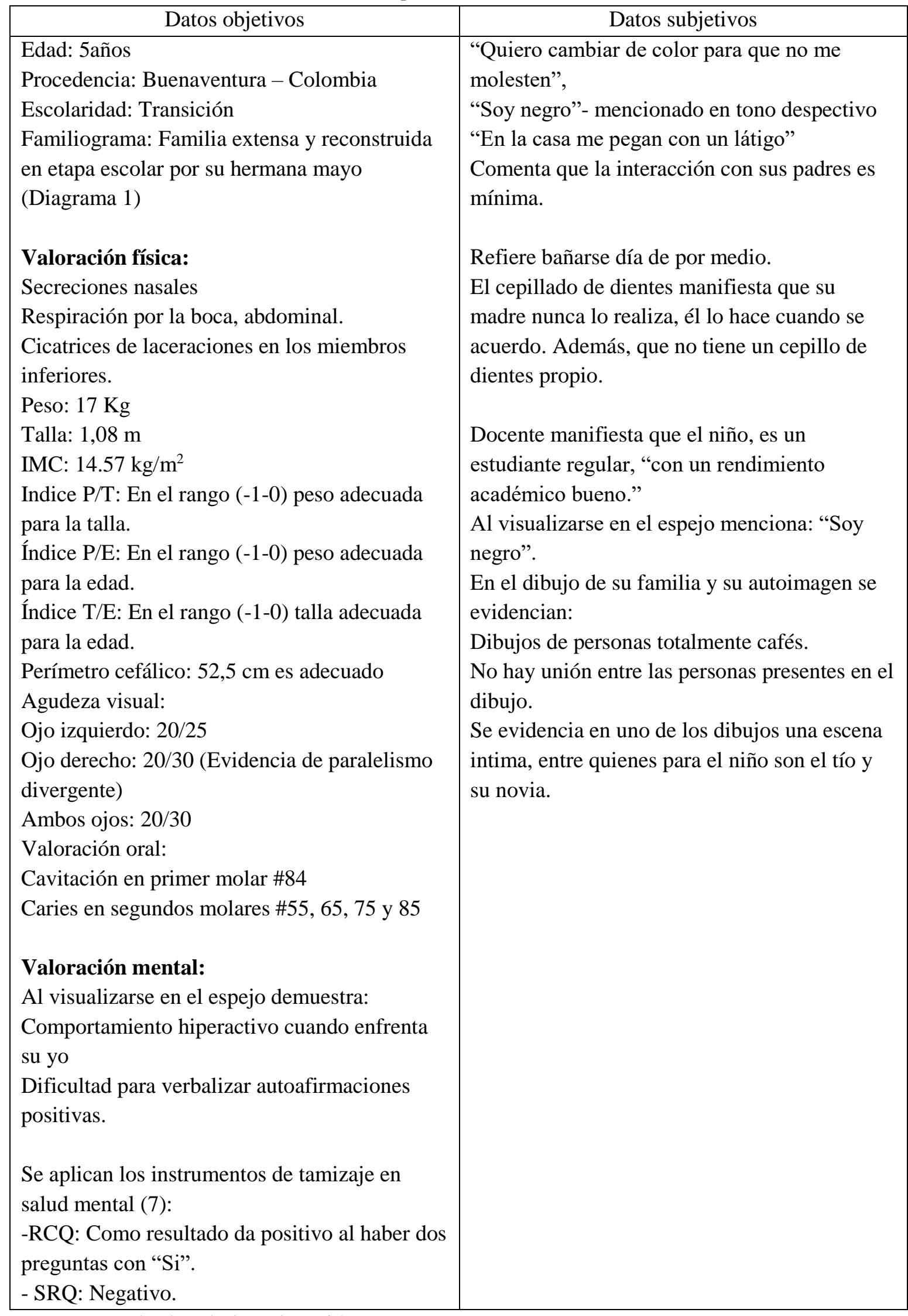

Fuente: Resultados de la valoración

2018, Horiz. Enferm., 28,3,238-250 
Diagrama 1. Familiograma y psicograma. Familia extensa reconstruida en etapa escolar. Fuente: Resultado de la valoración.

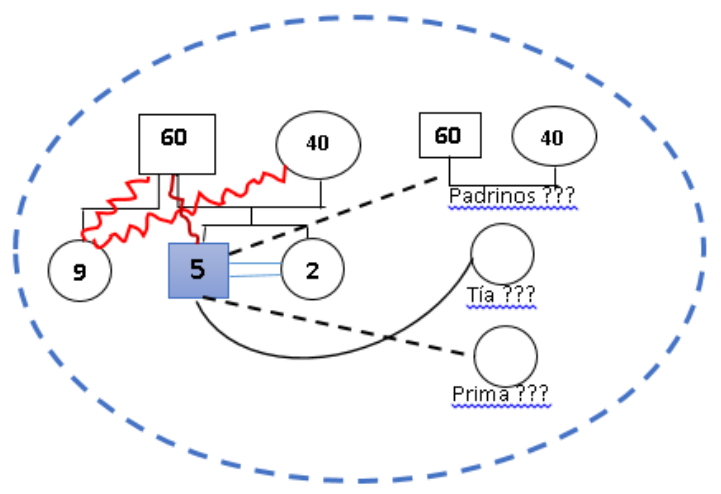

Diagrama 2. Inferencia diagnósticos de enfermería. Fuente: ${ }^{8,9,10,11,12,13)}$

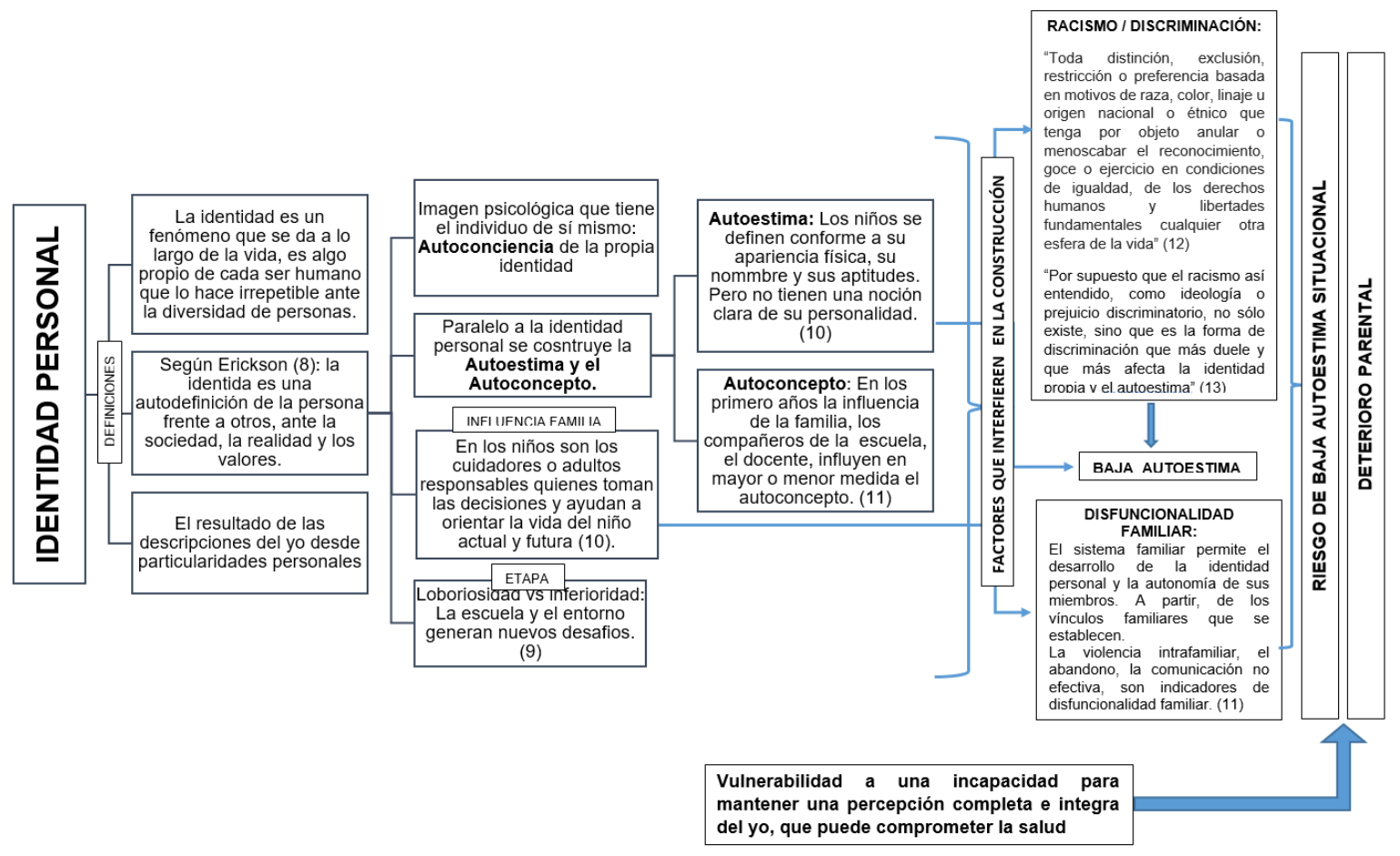

Los anteriores diagnósticos son seleccionados bajo las siguientes características definitorias relacionadas con autoestima baja, discriminación, etapa del crecimiento, procesos familiares disfuncionales, incongruencias culturales y percepción de prejuicios. Soportado el análisis por la TRM de Afrontamiento donde el modo de autoconcepto y dominio del rol están dificultando el proceso de afrontamiento; dentro del primer modo la autoestima y la identidad personal y física son los más afectados, y para el segundo la interacción con otros en condiciones 
Teoría de rango medio de enfermería para....

específicas, la cual no fluye de manera adecuada.

\section{Intervenciones de enfermería enfocadas} a iniciar un proceso de afrontamiento

La TRM de Afrontamiento de Roy $^{(5)}$ y la TRM de Adaptación a Eventos Difíciles de la Vida de Callista Roy ${ }^{(14)}$ generan a partir de la práctica intervenciones específicas para producir afrontamiento y adaptación en el individuo, su aplicación en este proceso de enfermería fue pertinente para trabajar los diagnósticos de enfermería centrales identificados, y una excelente estrategia para operacionalizar las intervenciones propuestas por la NIC.

La intervención central a trabajar recomendada por las dos TRM mencionadas anteriormente fue el humor y el control del estado de ánimo, tomadas como recurso para operacionalizar la NIC. En varias investigaciones se ha usado el humor como una estrategia de afrontamiento ya que permite enfrentar los eventos difíciles de la vida desde una mirada diferente, y juega un papel importante en la espiritualidad y el sentido de la vida del individuo ${ }^{(15)}$. De la misma forma se recomiendan sesiones de 15 a 20 minutos diarios de humor y risa ${ }^{(16)}$, ya que la risa puede actuar como mecanismo de defensa para reducir el estrés, potencializar la autoestima y mejorar los síntomas psicológicos relacionados con los sucesos negativos de la vida ${ }^{(15)}$. Es así como, el humor se relaciona con el aumento de la autoestima y la disminución de características depresivas en la personalidad $^{(15)}$. Las actividades realizadas usando el humor como intermediario, fueron ejercicios grupales para aumentar el soporte social y escolar. El Dr. Rod Martin menciona que "el humor es una actividad social, ya que desde la perspectiva evolutiva el humor nace como mecanismo para mejorar la cohesión del grupo"(17).

En contexto con la valoración, se relaciona el castigo físico con problemas de comportamiento y el castigo verbal con déficit en el autoconcepto, dejando secuelas importantes en la forma de afrontar de los niños en eventos difíciles de la vida posteriores a su infancia ${ }^{(18)}$. Campbell en sus investigaciones postula que los niños preescolares generan dos series de comportamiento frente al maltrato severo, estos pueden ser la internalización como la depresión y la externalización como la agresión ${ }^{(19)}$. Por lo anterior, se sugiere como intervención el control del estado de ánimo, donde la principal actividad es la valoración a profundidad del mismo. Investigaciones sugieren que este tipo de valoraciones se puede realizar en compañía de los padres, donde se pretende explorar el concepto de temperamento en ambas partes, y de esta forma facilitar las competencias y habilidades de crianza y reducir sustancialmente los problemas de comportamiento y de depresión en el preescolar $^{(20)}$. Además, retomando la intervención familiar, con actividades que estimulen la interacción familiar, el mantenimiento de los procesos familiares, habilidades para la vida ${ }^{(21)}$ utilizando la terapia grupal donde se integre toda la familia.

Finalmente, como se mencionó en la situación de enfermería la enfermera en formación se identificó con la persona cuidada, principalmente en las dificultades 
con el autoconcepto y la autoimagen, lo cual hizo que ella buscara ayuda en sesiones individuales con salud mental de enfermería para estar saludable mentalmente y de esta forma poder ofrecer cuidado de calidad.

\section{RESULTADOS ESPERADOS}

Los resultados esperados se plantean a partir del uso de la TRM de Afrontamiento y los indicadores ofrecidos por la Clasificación de Resultados de Enfermería (NOC), teniendo claro que el proceso de afrontamiento y posteriormente de adaptación a los cambios de la vida no se logra en una o dos sesiones con la persona cuidada, por ello, se realizó la remisión a salud mental para dar continuidad al proceso y lograr la meta de enfermería que para este caso en particular es generar el afrontamiento.

Se eligió como primera etiqueta la autoestima, que la NOC la defina como "el juicio personal sobre la capacidad de uno mismo"(22), con tres indicadores: Descripción del éxito en la escuela, descripción del éxito de compartir con sus iguales y mantenimiento del autocuidado/ higiene personal. Estos indicadores fueron el inicio de la potencialización de la autoestima y directamente del modo de autopercepción, generando herramientas efectivas para fortalecer y afianzar estrategias de afrontamiento. La siguiente etiqueta fue disminución de la ansiedad, definida como "Minimizar la aprensión, temor, presagios o inquietud relacionado con una fuente no identificada de peligro previsto"(22). Se seleccionó con la intensión de explorar más allá de lo ofrecido por la valoración inicial, para determinar el estado de ánimo del niño y su relación con la ansiedad, con la sospecha del uso de la felicidad y la personalidad extrovertida como una forma de negación ante su situación particular vivida en la escuela y el hogar. Posterior a lo anterior se eligieron tres indicadores que van de la mano del proceso de adaptación los cuales fueron: baja autoestima, estado de ánimo, tristeza. Haciendo claro énfasis que este resultado esperado sólo se obtendrá posterior a un proceso de valoración específico en el que se logre identificar el nivel de ansiedad a trabajar.

Estos resultados esperados sólo se verán reflejados en un trabajo constante con la persona cuidada, y la meta de enfermería se alcanzará, entendiendo que el fortalecimiento y afianzamiento de estrategias de afrontamiento efectivas es un proceso que se inicia solucionando los problemas en cada modo de afrontamiento planteado por la TRM de Afrontamiento, de esta manera la adaptación a los cambios diarios se hará sin complicaciones.

\section{CONCLUSIONES Y RECOMENDACIONES}

De la situación de enfermería acompañado por la narrativa "Un problema con el espejo", podemos concluir diferentes aspectos a nivel profesional, disciplinar y personal.

- Usar una TRM de enfermería fue indispensable para evidenciar que el proceso de enfermería es dinámico, autónomo y fundamentado. Esta aclaración se realiza con el fin de incentivar el uso de las teorías de enfermería en la práctica tanto hospitalaria como comunitaria, ya que guía el proceso 
Teoría de rango medio de enfermería para....

de intervenciones, planes de cuidado formulados y resultados esperados.

- Los objetivos a corto plazo propuestos con esta persona cuidada se cumplen, sin embargo, el trabajo a largo plazo es interrumpido por dinámica particulares del sitio de práctica y el constante movimiento de la familia, lo cual no permite que se realice un acompañando y seguimiento total al niño de su proceso de desarrollo y crecimiento, impidiendo la visualización amplia de los resultados a través de intervenciones propuesta.

- Es importante mencionar que, en casos de violencia familiar y discriminación, específicamente en menores de edad, el trabajo de la mano con la familia es fundamental para fortalecer factores protectores identificados y disminuir la vulnerabilidad al trabajar con los factores de riesgo, sin embargo, para el caso presente, la intervención con la familia se dificulta porque no se hace visible ni en la escuela ni en las citaciones previas.

- El seguimiento al caso se dio a través de la remisión a salud mental, donde es importante resaltar que cuando se usa una teoría como la TRM de Afrontamiento el caso requerirá de acompañamiento constante. En este caso el intervenir en un niño de cinco años será el punto clave para que sus procesos de cambios posteriores como la adolescencia y la adultez sean afrontados de manera eficaz y la adaptación se realice de forma fluida y sin grandes afectaciones en la salud mental.

- Para finalizar, se quiere resaltar que la enfermera que valoró y acompañó el proceso hasta la remisión, al identificarse con la persona cuidada y sus problemas con el espejo, decide cuidarse para poder dar cuidado de calidad. La TRM de 2018, Horiz. Enferm., 28,3,238-250
Afrontamiento de Roy señala que la enfermera en el proceso de velar por la salud de los demás, también debe generar autocuidado. Desde lo anterior se invita y recomienda a cada profesional en formación o en ejercicio, que el cuidado se da de calidad cuando la promoción y prevención inicia internamente.

\section{REFERENCIAS BIBLIOGRÁFICAS}

1. Panel on Research on Child Abuse and Neglect, National Research Council. Understanding child abuse and neglect. The National Academies Press, 1993.

2. De Bellis. The psychobiology of neglect. Child Maltreatment 2005; 10:150-72.

3. Díaz de Flores L, de Villalobos D, Mercedes M, Gallego de Pardo P, Gómez Daza B, Gómez de Obando, et al. Análisis de los conceptos del modelo de adaptación de Callista Roy. Aquichan, 2002; 2(1), p. 19-23.

4. Chinn PL, Jacobs MK, Huether SE. Theory and nursing: A systematic approach. St. Louis: The CV. Mosby Company. 2a. ed.; 1978.

5. Roy Callista. Generating Middle Range Theory. From evidence to practice. New York. Springer Publishing Company. 2014. p. 212.

6. Roy C., y col. El modelo de adaptación de Callista Roy, Editorial Appleton y Lange, $2^{\text {a }}$. ed. p. 32, 1999.

7. Childhood mental disorders in primary health care: results of observations in four developing countries. A report from the WHO collaborative Study on Strategies for Extending Mental Health Care Pediatrics. 1985; 68: 677-683 
8. Erikson, E. H. Identity: Youth and crisis (No. 7). WW Norton \& Company; 1994.

9. Erikson, E. H. Childhood and society. WW Norton \& Company; 1993.

10. Gall, O. Identidad, exclusión y racismo: reflexiones teóricas y sobre México. Rev Mex de Sociol. 2004; 66(2): 221-259.

11. Herrera, P. M. La familia funcional y disfuncional, un indicador de salud. Revista cubana de medicina general integral. $\quad 1997 ; 13(6)$ : 591-595. Revisado en: http://scielo.sld.cu/scielo.php?script=s ci_arttext\&pid=S086421251997000600013

12. Cisneros, I. H. Formas modernas de la intolerancia: De la discriminación al genocidio. México; 2005.

13. Gall, O. Racismo, mestizaje y modernidad: visiones desde latitudes diversas. México; 2007.

14. Roy C. Generating Middle Range Theory. From evidence to practice. New York. Springer Publishing Company. 2014. p. 238-247.

15. Bennett MP, Lengacher C. Humor and Laughter May Influence Health: II. Complementary Therapies and Humor in a Clinical Population. EvidenceBased Complementary and Alternative Medicine, 2006, 3(2), 187-190. http://doi.org/10.1093/ecam/nel014
16. Friedman HS, Tucker JS, TomlinsonKeasey C, Schwartz JE, Wingard DL, Criqui MH. Does childhood personality predict longevity?. Journal of Personality and Social Psychology, 1993, 65(1), $176 \quad-\quad 185$. http://dx.doi.org/10.1037/00223514.65.1.176

17. Martin R, Kuiper NA. Three Decades Investigating Humor and Laughter: An Interview With Professor Rod Martin. Europe's Journal of Psychology, 2016, 12(3), 498-512. http://doi.org/10.5964/ejop.v12i3.1119

18. Berzenski SR, Yates TM. Preschoolers' Emotion Knowledge and the Differential Effects of Harsh Punishment. Journal of Family Psychology: JFP : Journal of the Division of Family Psychology of the American Psychological Association (Division 43), 2013, 27(3), 463-472. http://doi.org/10.1037/a0032910

19. Campbell S. Behavior problems in preschool children: A review of recent research. Journal of Child Psychology and Psychiatry. 1995, 36, 113-149.

20. Roy C. Predicting school-age behavior problems: the role of early childhood risk factors. Pediatr Nurs Subsets: Nursing, 2008, 34 (1), 37-44.

21. Baca, M.E. Evidencias en prevención de la violencia que afecta niños, niñas y adolescentes. Washington, D,C.: OPS, 2013. 